\title{
Exploiting Length-Dependent Effects for the Design of Single-Material Systems with Enhanced Thermal Transport Properties
}

\author{
Jean-Baptiste Bouquet, Frederic Burgaud, Julian J. Rimoli* \\ School of Aerospace Engineering, Georgia Institute of Technology \\ Atlanta GA 30332
}

\begin{abstract}
Topology optimization approaches for thermal transport problems generally rely on the presence of high contrast in thermal conductivity between constituent materials. Even though this might result in topological configurations that are optimal according to some desired thermal metric, the resulting systems might be impractical from a mechanical standpoint: high contrast in thermal conductivity generally translates into disparate ranges of thermal expansion, which in turn may lead to mechanical failure. In this paper, we focus on the combination of multi-scale modeling and topology optimization techniques for the design of polycrystalline single-material systems with enhanced thermal transport properties. Spatial variation of the length-dependent thermal conductivity is achieved by grading the characteristic grain size of the microstructure. We adopt a multi-scale approach to compute the attainable range of thermal conductivity due to grain-size effect, given maximum and minimum grain sizes and an operating temperature range. Then, we utilize an adaptive topology optimization technique to obtain an optimal grain size distribution, while accounting for manufacturing constraints. Through a series of specific examples, we demonstrate the extent to which thermal transport properties can be optimized without resorting to the adoption of multi-material systems.
\end{abstract}

Keywords: Heat transport, topology optimization, size effects, multi-scale modeling

\section{Introduction}

Topology optimization is a mathematical technique aimed at determining material distribution within an engineering component. The goal of this method is that, for certain

*Corresponding author. E-mail: rimoli@gatech.edu

Preprint submitted to Elsevier

May 26, 2016 


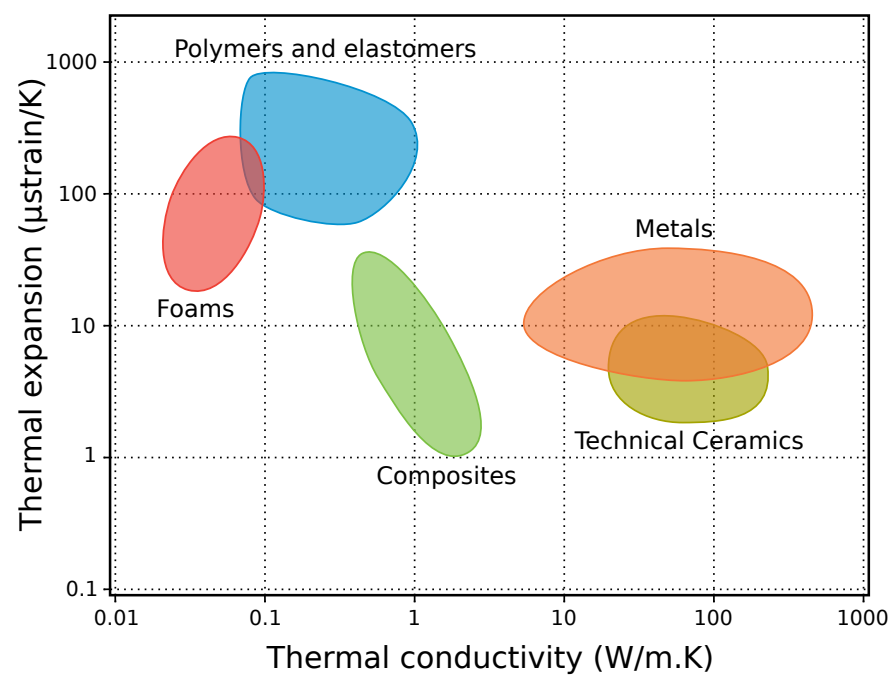

Figure 1: Ashby graph of thermal conductivity vs. thermal expansion coefficient for various material systems.

boundary conditions and under specific constraints, the component performs optimally in the sense of a given metric. Early works on topology optimization focused on mechanical performance of structures and date back to the beginning of the past century, although the field did not reach its full potential until the seminal contribution of Bendsoe and Kikuchi [1], which led to the development of practical and efficient numerical schemes.

Due to the direct analogy between mechanical and thermal problems, topology optimization techniques have subsequently been applied to a rather large variety of heat transfer problems. In this area, the objective is generally to distribute a limited amount of conductive materials within the analysis domain in order to optimize the thermal performance of the component. Common design goals include minimizing the mean temperature over the domain [2, 3] and maximizing the temperature diffusivity of a design [4], to name a few.

Over the past two decades, various optimization methods have been developed in order to solve increasingly complex problems, as well as to improve the robustness of the obtained results. Among them, some prominent approaches include: homogenization method [5], penalized topology optimization [6, 7], evolutionary structural optimization [8], level setbased topology optimization [9], and discrete material optimization [10]. A common feature of all of these approaches is that designs are based on multi-phase systems, which generally require high contrast material properties between constituents. Even though this might result in topological configurations that are optimal according to some desired thermal metric, resulting configurations might be impractical from a mechanical standpoint. 
As Fig. 1 shows, a high contrast in the value of thermal conductivity between materials is generally associated with disparate values on the corresponding thermal expansion coefficients. This implies that, as the temperature of a system departs from a stress-free configuration, large thermal stresses develop at the interfaces between constituent materials. This, in turn, could lead to premature thermo-mechanical failure. Ideally, we would like to be able to optimize the thermal properties of components without compromising their mechanical integrity.

In this paper, we investigate the possibility of circumventing the aforementioned limitation by optimizing the spatial grain size distribution on single-material polycrystalline systems: it is well known that the effective thermal conductivity of a material decreases drastically as the characteristic length scale of its microstructure is reduced. Recent investigations by Bouquet and Rimoli [11] show that, by taking advantage of this effect, one could tailor the microstructure of a material, e.g. through grading of the grain size, and affect its internal temperature distribution. We combine their concurrent multi-scale model with topology optimization techniques to develop single-material systems with optimized thermal transport properties.

The rest of the article is organized as follows. In Section 2, we outline the fundamentals of our multi-scale model and introduce the adopted optimization methods. In Section 3, we present key optimization results to illustrate the capabilities of the proposed approach. Finally, in Section 4 we draw the conclusions of this paper and discuss future research directions.

\section{Concepts and methods}

In this work, we depart from previous approaches to topology optimization in thermal transport problems by considering systems where the thermal conductivity can vary continuously within an attainable range. This variation of thermal conductivity is achieved by varying the internal characteristic length of the material under consideration, as it will be explained in the following section. This approach allows us to optimize single-material systems, thus avoiding mechanical failure issues resulting from previously mentioned thermal expansion coefficient mismatch between different materials.

In our approach, we first compute the attainable range of thermal conductivity by following a model recently introduced by Bouquet and Rimoli [11. Then, by assuming separation of scales, we optimize a material system for a continuous distribution of thermal conductivity constrained by the previously computed attainable range. Finally, the separation of scales assumption is verified by reconstructing the optimized microstructure, computing the value of the objective function, and comparing it with the value obtained for the optimized continuous system. The overall multi-scale optimization procedure is depicted schematically in Fig. 2. 


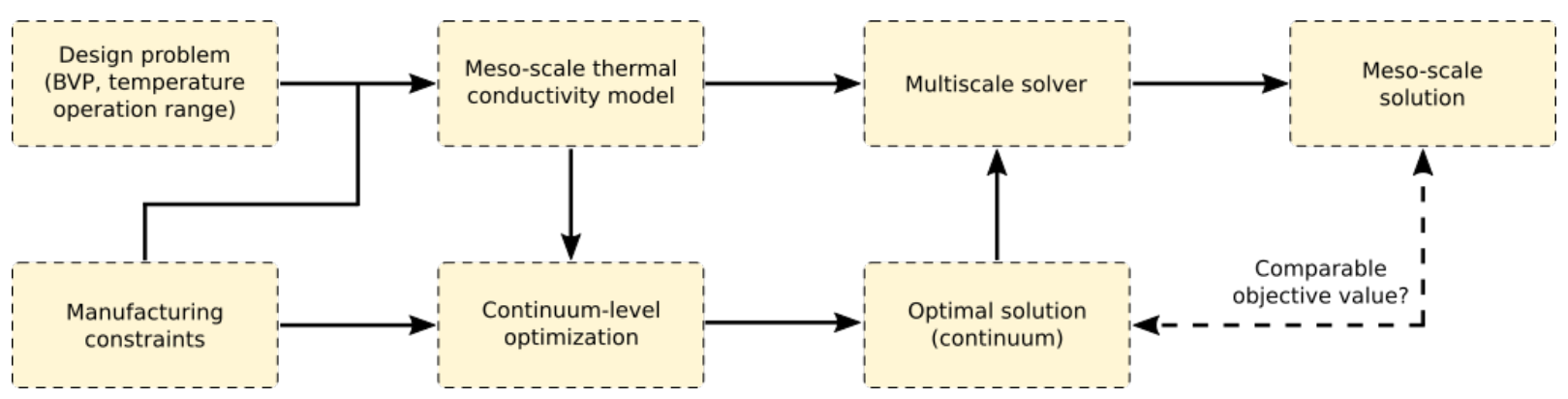

Figure 2: Schematics of overall multi-scale optimization process.

In the following subsections, we first describe the multi-scale model that allows us to compute the attainable range of thermal conductivity, then formulate our approach to the optimization problem, and finally detail the procedure to verify the separation of scales assumption.

\subsection{Multi-scale heat analysis}

As the characteristic length scale of a material is reduced, e.g. due to the presence of microstructural interfaces, its effective thermal conductivity decreases drastically [12, [13, 14, 15]. In materials where heat conduction is mediated mainly by phonons, for example ceramics, this reduction is primarily due to the shortening of the phonon mean free paths [12, 13, 16, 17] as a result of scattering at the interfaces [18, 19]. In order to account for this effect, Bouquet and Rimoli [11] recently developed a multi-scale framework consisting of: (i) a sub-micron scale model for the thermal conductivity based on the Boltzmann transport equation under the relaxation time approximation, and (ii) a classic Fourier heat transport model at the mesoscale that explicitly resolves the microscopic geometric features of the material. In this work, we utilize their methodology first to estimate an attainable range of thermal conductivity based on the expected range of grain size and temperature operation of the device to optimize, and then to reconstruct the microstructure of the resulting optimized system. For the sake of completeness, this methodology is briefly summarized in the following subsections.

\subsubsection{Bulk thermal conductivity}

In materials where heat transfer is dominated by phonon transport, the bulk thermal conductivity can be estimated from the kinetic theory using the Boltzmann transport equation under the relaxation time approximation. This approach represents a particlelike view of heat transfer and accounts for both diffuse and ballistic transport effects. The 
bulk thermal conductivity $D$ is calculated using the well-known formula [18, 20]

$$
D=\frac{k_{b}}{(2 \Pi)^{3}} \sum_{P=L, T} \int_{B Z} \tau(\vec{k}) v_{g}^{2}(\vec{k}) \cos ^{2} \alpha\left(\frac{\hbar \omega(\vec{k})}{k_{b} T}\right)^{2} \frac{\exp \left(\hbar \omega(\vec{k}) / k_{b} T\right)}{\left[1-\exp \left(\hbar \omega(\vec{k}) / k_{b} T\right)\right]^{2}} d \vec{k}
$$

where $k_{b}$ and $\hbar$ are respectively the Boltzmann and the reduced Planck constants, $P$ is the polarization of the phonons, $\tau$ is the effective relaxation time, $\omega$ is the phonon frequency, $T$ is the temperature, $v_{g}$ is the phonon group velocity and $\alpha$ is the angle between the thermal gradient and the wavevector $\vec{k}$. The variable of integration $d \vec{k}$ is the elementary volume in the $\mathrm{k}$-space and the integration is performed over the first Brillouin zone (BZ) of the lattice. This prediction of the thermal conductivity incorporates the contribution of both longitudinal and transverse acoustic phonons. We adopt the common assumption that the optical phonons do not contribute to the energy transport due to their slow group velocity [20, 21, 22]. Thus, we do not include them into the current calculation of the thermal conductivity. The bulk effective relaxation time $\tau(\vec{k})$ represents the average characteristic time for a phonon to undergo two consecutive scatterings and accounts for all the different scattering processes encountered in the model. Considering the scattering processes to be independent from each other, the Matthiessen's rule yields to [23]

$$
\tau^{-1}=\tau_{U}^{-1}+\tau_{I}^{-1}+\tau_{C}^{-1}
$$

where $\tau_{U}, \tau_{I}$ and $\tau_{B}$ are the relaxation times respectively associated with Umklapp, phonon isotope and Casimir limit scatterings. More precisely, the Umklapp scattering models the phonon-phonon collisions, the phonon isotope scattering represents the collisions between phonons and impurities inside the crystal, and the Casimir scattering is the low temperature limit, constraining the prominent enlargement of the phonon mean free path. Their expressions are given by

$$
\begin{aligned}
& \tau_{U}^{-1}=A_{1, P} \omega^{2} T \exp (-b / T) \\
& \tau_{I}^{-1}=A_{2} \omega^{4} \\
& \tau_{C}^{-1}=A_{3}
\end{aligned}
$$

where constants $A_{1, P}, A_{2}, A_{3}$, and $b$ must be determined by calibrating the model with experimental values of the bulk thermal conductivity.

In sample problems, we will focus on systems composed of polycrystalline silicon, due to the abundance of data regarding its material properties and size effects. Silicon is assumed to be isotropic in the k-space, and adopted dispersion relations are those of bulk silicon along the [100]-direction. Group and phase velocities are derived from measured dispersion relations [24, 25] and tabulated for each wavevector. The remaining constants are fitted by assuming that $80 \%$ of the heat transport occurs through transverse phonons, resulting in the values listed in Table 1 . 


\begin{tabular}{|c|c|}
\hline Constant & Value \\
\hline$A_{1, L}$ & $3.28 \times 10^{-19}$ s.K $K^{-2}$ \\
$A_{1, T}$ & $0.69 \times 10^{-19}$ s. $K^{-2}$ \\
$A_{2}$ & $2.5 \times 10^{-45} s^{3}$ \\
$A_{3}$ & $6.18 \times 10^{5} s^{-1}$ \\
$b$ & $140 \mathrm{~K}$ \\
\hline
\end{tabular}

Table 1: Material constants for bulk silicon.

\subsubsection{Length-scale effect}

Eq. 11 provides the expression to compute the coarse grain thermal conductivity of a given material. However, the thermal conductivity is a size-dependent property. As the characteristic length scale of the material is reduced, its effective thermal conductivity decreases drastically [12, 13, 16]. In the case of polycrystalline materials, the thermal conductivity is highly affected by the size of the constituting grains, as well as by their particular shape. This phenomenon is explained by the reduction of the phonon mean free path, which translates into a reduction of the relaxation time $\tau_{r}(\vec{k})$ such that

$$
\tau_{r}(\vec{k})=\tau(\vec{k}) g(\vec{k})
$$

where $g(\vec{k})$ is the size effect reduction function. The expression of $g(\vec{k})$ has been extensively developed by Turney et al. [26], and is formulated more conveniently in the case of micrograins as

$$
g(\vec{k})=1-\frac{(1-p) \exp \left(-\delta_{1}(\vec{k}) / \Lambda(\vec{k})\right)}{1-p \exp \left(-\delta_{2}(\vec{k}) / \Lambda(\vec{k})\right)}
$$

where $\delta_{1}$ and $\delta_{2}$ are the distances to the boundaries of the domain as depicted in Fig. 3 and depend on the direction of the wavevector $\vec{k}$.

By inserting the reduced relaxation time into Eq. 1, one can compute the reduced thermal conductivity $D_{\mathrm{r}}$ at a specific point within the grain. That is, by following this approach, the thermal conductivity is a locally defined quantity that varies within the domain of each grain. For the purposes of this work, we assume separation of scales and compute the effective thermal conductivity of a given grain as

$$
D_{\text {eff }}=\frac{1}{A_{\Omega_{g}}} \int_{\Omega_{g}} D_{\mathrm{r}} d A
$$

where $\Omega_{g}$ is the domain of the grain, $A_{\Omega_{g}}$ its area, and $D_{\mathrm{r}}$ the value of the reduced thermal conductivity at a point inside the domain, as previously defined. Following this procedure, 

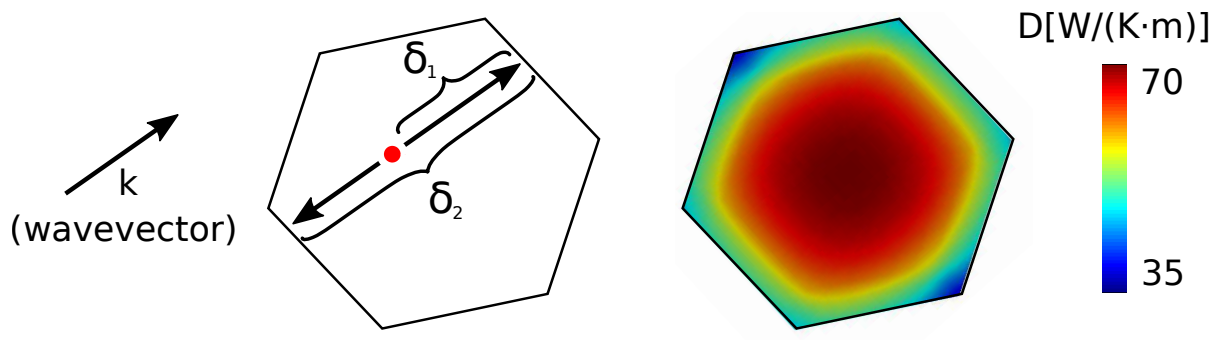

Figure 3: Schematics depicting the relevant distances in the reduction function, and the resulting reduced thermal conductivity $D_{\mathrm{r}}$ within a grain. The effective thermal conductivity $D_{\text {eff }}$ for a grain of a given size is the area average of this quantity.

it is possible to compute the effective thermal conductivity of grains of arbitrary size, at arbitrary temperature.

\subsubsection{Continuum-level heat analysis}

A key component of our approach is that we assume separation of scales. That is, we assume that at the continuum level, the thermal conductivity of the material is a locally (point-wise) defined quantity that depends on the temperature at that point and the characteristic grain size of the microstructure at the same location. In this way, we can model a continuum as a material obeying Fourier's heat equation, with the consideration that thermal conductivity is no longer a constant quantity. Consequently, the heat transport problem on a domain $\Omega$ is modeled by the heat equation

$$
\frac{\partial}{\partial x^{i}}\left(\frac{D_{\mathrm{eff}}(T)}{\rho C_{p}} \frac{\partial T}{\partial x^{i}}\right)=\frac{\partial T}{\partial t} \quad \text { in } \Omega
$$

where $T$ is the temperature in an orthonormal basis with coordinates $x^{i}, D_{\text {eff }}(T)$ is the temperature-dependent effective thermal conductivity, $\rho$ is the mass density and $C_{p}$ is the specific heat capacity. The corresponding boundary conditions are given by

$$
T=\bar{T} \quad \text { on } \partial \Omega_{1} \quad q^{i}=\bar{q}^{i} \quad \text { on } \partial \Omega_{2}
$$

where $q^{i}$ represents the components of the heat flux vector expressed in an orthonormal basis with coordinates $x^{i}$, and $\partial \Omega_{1}$ and $\partial \Omega_{2}$ must satisfy

$$
\partial \Omega_{1} \cup \partial \Omega_{2}=\partial \Omega, \quad \partial \Omega_{1} \cap \partial \Omega_{2}=\emptyset
$$

To compute the heat transfer problem on arbitrary domains, the diffusion problem can be expressed in weak form and subsequently discretized by means of a finite element scheme, resulting in

$$
M_{a b} \frac{\partial \hat{T}_{b}}{\partial t}+K_{a b} \hat{T}_{b}=Q_{a}
$$


with

$$
M_{a b}=\int_{\Omega} \rho C_{p} N_{a} N_{b} d \Omega \quad K_{a b}=\int_{\Omega} \frac{\partial N_{a}}{\partial x^{i}} D_{\mathrm{eff}} \frac{\partial N_{b}}{\partial x^{j}} d \Omega \quad Q_{a}=-\int_{\partial \Omega_{2}} N_{a} N_{b} \hat{\bar{q}}_{b}^{i} n_{i} d S
$$

where $\hat{T}_{b}$ is the temperature at node $b, \hat{\bar{q}}_{b}^{i}$ are the components of the heat flux vector at node $b, n_{i}$ are the components of the unit normal to the surface $\Omega_{2}$, and $N_{i}$ are the interpolation or shape functions. The terms $M_{a b}$ and $K_{a b}$ are called the mass and stiffness matrices respectively by analogy with solid mechanics problems, and $Q_{a}$ is called the forcing term. The integrals in Eqs. 13 are evaluated through a quadrature scheme, and the resulting differential Eq. 12 is usually integrated in time by means of a forward Euler procedure. As this study focuses on steady-state problems, the temporal derivative vanishes and Eq. 12 then reduces to

$$
K_{a b} \hat{T}_{b}=Q_{a}
$$

which can be solved by inverting the matrix determined by $K_{a b}$.

\subsection{Optimization methodology}

In the previous subsections, we illustrated how it is possible to compute the variation of the thermal conductivity of a given material due to changes in its characteristic length scale. This, combined with the separation of scales assumption, allows us to perform topology optimization on a thermal system for a continuous distribution of thermal conductivity. This fact, by itself, constitutes a departure from traditional approaches to topology optimization of thermal systems, which generally assume multi-material configurations and the consequent discreteness of the available values of thermal conductivity.

Using our proposed methodology, we can translate manufacturing constraints (range of grain size) and expected operating conditions (range of temperature) into an attainable range of thermal conductivity $\left[D_{\min }, D_{\max }\right]$. Then, using this range as a constraint, a given objective function could be minimized over the spatial distribution of thermal conductivity for the system under consideration. Commonly adopted objective functions aim at: (i) minimizing the average temperature in the material [27], or (ii) maximizing its thermal diffusivity [4]. Other objective functions may be defined as well.

In this paper, optimization is achieved through the use of the genetic algorithm, which is arguably the most commonly used evolutionary algorithm and one of the most conventional non-gradient methods [28, 29]. The main drawback of this approach is the absence of mathematical convergence theory and thus no assurance of optimality of the final solution found. While we acknowledge that there might be better optimization approaches for the problem under consideration, it is not our objective to solve it in the best or most efficient manner. In this work, we intend to demonstrate the possibility of optimizing thermal 
transport properties through single-material systems, by means of combining multi-scale and topology optimization techniques.

In our approach, the geometry of the system under consideration is discretized using a triangular mesh consisting of $N$ nodes. Design variables, e.g., thermal conductivity, are assigned to each node on the mesh. We denote $D_{d}$ the value of the effective thermal conductivity at a node. Finite element calculations are performed over the same mesh to evaluate the objective function $f\left(D_{d}\right)$, with the values of the thermal conductivity at quadrature points of the finite elements being obtained through interpolation from the design points. The resulting optimization problem is then written as

$$
\begin{array}{cl}
\underset{D_{d}}{\operatorname{minimize}} & f\left(D_{d}\right) \\
\text { subject to } & g\left(D_{d}\right) \leq 0 \\
& D_{d} \in\left[D_{\min }, D_{\max }\right]^{N}
\end{array}
$$

where $g\left(D_{d}\right) \leq 0$ represents any other additional constraints, e.g., average thermal conductivity over the domain.

By using this procedure, the smoothness and resolution of the optimal solution depend on the geometry of the mesh, as it will be depicted in a sample problem below. Briefly, we can say that highly regular meshes constraint the optimal solution to have the same underlying structure. To alleviate this issue, we add to the optimization problem the coordinates of the control points $X_{d}$. That is, optimization of the objective function is performed over the distribution of thermal conductivity and location of the control points. This strategy resembles r-adaption schemes adopted for finite element problems in elastostatics [30], resulting in the modified optimization problem

$$
\begin{array}{cl}
\underset{X_{d}, D_{d}}{\operatorname{minimize}} & f\left(X_{d}, D_{d}\right) \\
\text { subject to } & g\left(X_{d}, D_{d}\right) \leq 0 \\
& X_{d} \in \Omega^{N} \\
& D_{d} \in\left[D_{\min }, D_{\max }\right]^{N}
\end{array}
$$

To illustrate the effects of the mesh adaption technique, or lack thereof, we apply the previously described optimization method to a representative problem first introduced in [4], see Fig. 4. The analysis domain is a square of length $L_{s}=200 \mu m$, composed of two materials, one with thermal conductivity $D_{\max }=148 \mathrm{~W} \mathrm{~K}^{-1} \mathrm{~m}^{-1}$ and the other one with no ability to transfer heat at all. The bottom and top edges include heat sinks at $300 \mathrm{~K}$. The right and left edges are subjected to an incoming heat flux $q_{0}=353 \mathrm{Wm}^{-1}$. Both heat sinks and incoming flux are located at the center of their respective edges over $10 \%$ of the edge 


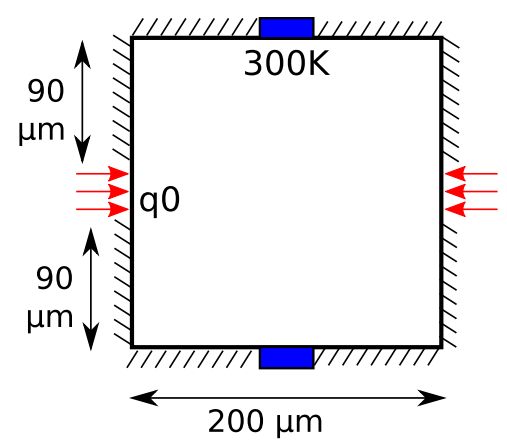

Figure 4: Schematics for optimization problem to be solved with and without mesh adaption.

length. In this problem, the goal is to find the distribution of thermal conductivity that maximizes the thermal diffusivity of the plate. This objective is achieved by minimizing the temperature at points $P_{1}$ and $P_{\mathrm{r}}$, located at the middle of the vertical edges. Due to the symmetry of this configuration, the related values $T_{\mathrm{Pl}}$ and $T_{\mathrm{Pr}}$ are identical. It is worth noting that an additional constraint on the average thermal conductivity $D_{\text {mean }}$ is added, such that $D_{\text {mean }}=45 \mathrm{WK}^{-1} \mathrm{~m}^{-1}$. Structurally, it means that $32.9 \%$ of the material has a thermal conductivity $D_{\max }=148 W^{-1} \mathrm{~m}^{-1}$, while the rest has a thermal conductivity $D_{\min }=0 W K^{-1} m^{-1}$.

The problem is solved using two approaches: with and without mesh adaption. The initial mesh for both problems is structured, given by a uniform distribution of design points over a square grid. For the problem without mesh adaption, the design variables are the thermal conductivity values $D_{d}$ at the design points. For the problem with mesh adaption, we optimize over thermal conductivity values $D_{d}$ and design point locations $X_{d}$. Notice that the addition of new design variables $X_{d}$ would result in a larger optimization problem if the number of design points is to be kept constant. Consequently, the problem with mesh adaption is optimized over fewer design points, maintaining the number of optimization variables for both problems identical, totaling 108 for each of them.

Under these assumptions, the optimization problem with fixed design points is expressed as

$$
\begin{array}{cl}
\underset{D_{d}}{\operatorname{minimize}} & \frac{T_{P l}\left(D_{d}\right)+T_{P r}\left(X_{d}, D_{d}\right)}{2} \\
\text { subject to } & \frac{1}{L_{s}^{2}} \int_{x \in\left[0, L_{s}\right]^{2}} D\left(x, D_{d}\right) d x \leq D_{\text {mean }} \\
& D_{d} \in\{0,148\}^{N}
\end{array}
$$




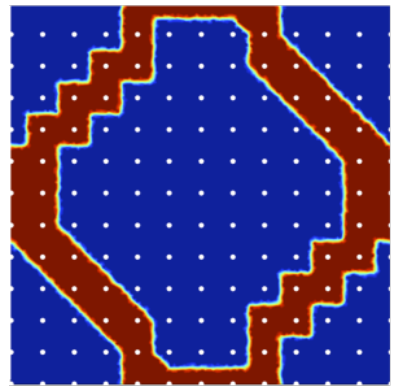

(a) Fixed design points

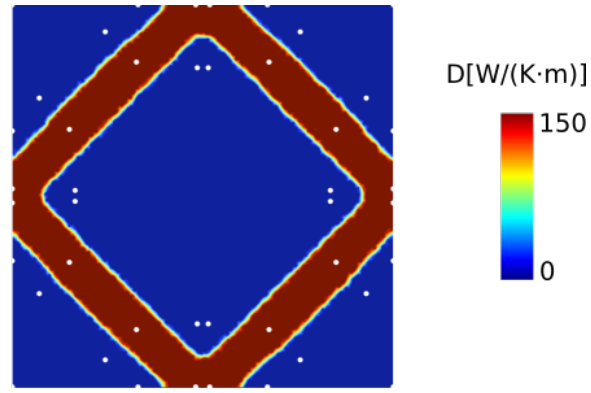

(b) Adaptive mesh

Figure 5: Optimization results with (right) and without (left) mesh adaption. Red color represents regions with high the thermal conductivity value, while blue color indicates regions with no thermal conductivity.

whereas for the adaptive problem we can write

$$
\begin{array}{cl}
\underset{X_{d}, D_{d}}{\operatorname{minimize}} & \frac{T_{P l}\left(X_{d}, D_{d}\right)+T_{P r}\left(X_{d}, D_{d}\right)}{2} \\
\text { subject to } & \frac{1}{L_{s}^{2}} \int_{x \in\left[0, L_{s}\right]^{2}} D\left(x, X_{d}, D_{d}\right) d x \leq D_{\text {mean }} \\
& X_{d} \in\left[0, L_{s}\right]^{2 N} \\
& D_{d} \in\{0,148\}^{N}
\end{array}
$$

The optimization results for the problem without adaption are shown in Fig. 5 a. As the thermal conductivity on the control points is interpolated over a regular mesh, the resulting optimal solution is highly structured, and reflects the underlying topology of the mesh.

In contrast, Fig. 5b shows the optimal solution for the problem with mesh adaption. Although giving mobility to the control points significantly increases the dimensionality of the problem, it gives more flexibility to the distribution of thermal conductivity, thus enabling more complex and smoother shapes with a lower amount of points. Consequently, the optimal design is less sensitive to the mesh. It can be observed that control points are more densely placed in areas with large variations in thermal conductivity distribution.

It is worth noting that the effect of adaptivity on the optimal value of the objective function is non-negligible. Indeed, with fixed design points the temperature at point $P$ is $T_{P}=397.61 \mathrm{~K}$, while with the adaptive design mesh it drops to $T_{P}=375.30 \mathrm{~K}$. Another benefit of allowing the mesh to adapt is that resulting smooth geometries are potentially easier to manufacture than the highly structured ones resulting from regular meshes. With a similar number of variables used in the algorithm, the fixed-point optimization results 


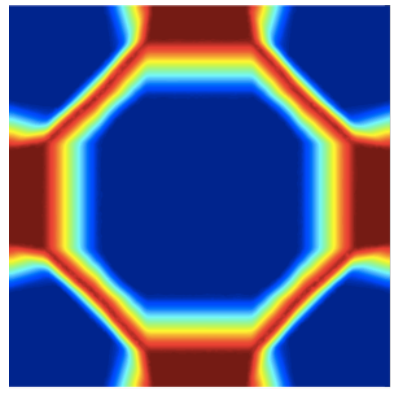

(a) Continuous optimization

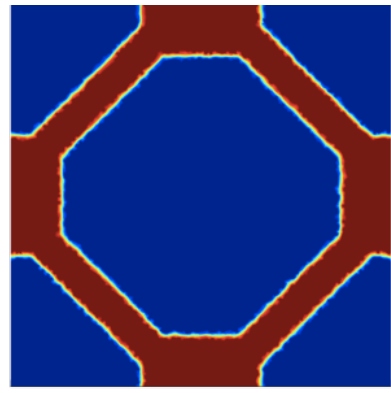

(b) Discrete optimization
$\mathrm{D}[\mathrm{W} /(\mathrm{K} \cdot \mathrm{m})]$

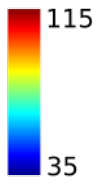

35

Figure 6: Comparison between discrete versus continuous optimization approaches.

in a suboptimal configuration due to the lack of freedom over the shape of the thermal conductivity distribution.

In the previous example, optimization was performed over a discrete range of thermal conductivity value for purposes of illustration. In contrast, we propose to perform such optimization on single material systems, resulting on a continuous range of attainable thermal conductivity which depends on some characteristic material length scale. Let us now consider the same problem as before, but restraining the thermal conductivity values to remain within reported values for nano- and micro-grained silicon: $D \in[40,110] W^{-1} \mathrm{~m}^{-1}$. Let us also assume that the average thermal conductivity on the domain is constrained to be $D_{\text {mean }}=61 \mathrm{WK}^{-1} \mathrm{~m}^{-1}$. This translates into the following formulation

$$
\begin{array}{cl}
\underset{X_{d}, D_{d}}{\operatorname{minimize}} & \frac{T_{P l}\left(X_{d}, D_{d}\right)+T_{P r}\left(X_{d}, D_{d}\right)}{2} \\
\text { subject to } & \frac{1}{L_{s}^{2}} \int_{x \in\left[0, L_{s}\right]^{2}} D\left(x, X_{d}, D_{d}\right) d x \leq D_{\text {mean }} \\
& X_{d} \in\left[0, L_{s}\right]^{2 N} \\
& D_{d} \in[40,110]^{N}
\end{array}
$$

The resulting optimal solution is visualized in Fig. 6a. On the other hand, Fig. 6b shows the optimal configuration for the equivalent discrete problem in which the thermal conductivity is forced to adopt the extreme values of the continuous range, while maintaining the same mean value constraint. The temperature attained at point $P$ is fairly similar for both cases: $T_{P}=371.93 \mathrm{~K}$ for the discrete configuration, and $T_{P}=371.23 \mathrm{~K}$ for the continuous one. This highlights the fact that solutions for this kind of optimization problems tend to be very sharp. This could be difficult to materialize from a fabrication perspective, as currently manufacturing techniques only allow for smooth transitions in the grain size distribution. 
The previous discussion motivates the introduction of an extra constraint on the model, which relates to the grain-size gradient. This restriction is enforced indirectly through a constraint on the thermal conductivity gradient. The latter gradient is calculated within each element and the maximum value throughout the domain $\nabla D_{\max }$ is bounded by $\nabla D_{\mathrm{opt}}$

$$
\nabla D_{\max } \leq \nabla D_{\mathrm{opt}}
$$

The calculation of $\nabla D_{\max }$ relies on the derivative of the shape functions $N_{i}(x, y)$ utilized to interpolate the design variables, such that

$$
\nabla D_{\max }=\max _{\text {elts }}\left\{\sqrt{\left(\sum_{i=1}^{3} \frac{\partial N_{i}(x, y)}{\partial x} D_{i}\right)^{2}+\left(\sum_{i=1}^{3} \frac{\partial N_{i}(x, y)}{\partial y} D_{i}\right)^{2}}\right\}
$$

where $D_{i}$ is the effective thermal conductivity at the $i^{\text {th }}$ node of the triangle.

The limiting thermal conductivity gradient $\nabla D_{\text {opt }}$ is defined as

$$
\nabla D_{\mathrm{opt}}=\gamma \frac{D_{\max }-D_{\min }}{L_{s}}
$$

where $D_{\max }$ and $D_{\min }$ represent the bounds of the thermal conductivity, and $L_{s}$ is the characteristic length of the analysis domain. Thus, the parameter $\gamma$ is non-dimensional and can be considered as a parameter of the system.

The methodology introduced thus far allows us to obtain, for a given metric and set of constraints, the optimal thermal conductivity distribution within a component. A key assumption throughout this process is that separation of scales holds true, that is, that the model could be optimized at the continuum scale adopting an attainable range of thermal conductivity computed at the mesoscale. The remaining task is then to verify whether or not this assumption holds true. In order to do so, we must generate an explicit reconstruction of the microstructure for the optimized system, and compute the objective function over that domain. This value, in turn, must be compared to its continuum counterpart. In the next section, we describe the procedure we utilize to generate an explicit reconstruction of the microstructure.

\subsection{Verification of separation of scales assumption}

By following the procedures described in subsections 2.1 and 2.2, we obtain an optimal distribution of thermal conductivity $D(X)$ over the analysis domain and the corresponding temperature field $T(X)$ for the optimal solution. The last step of our approach consists on the verification of the original separation of scales assumption. This is achieved by explicitly reconstructing the microstructure of the material from the optimization results, and 


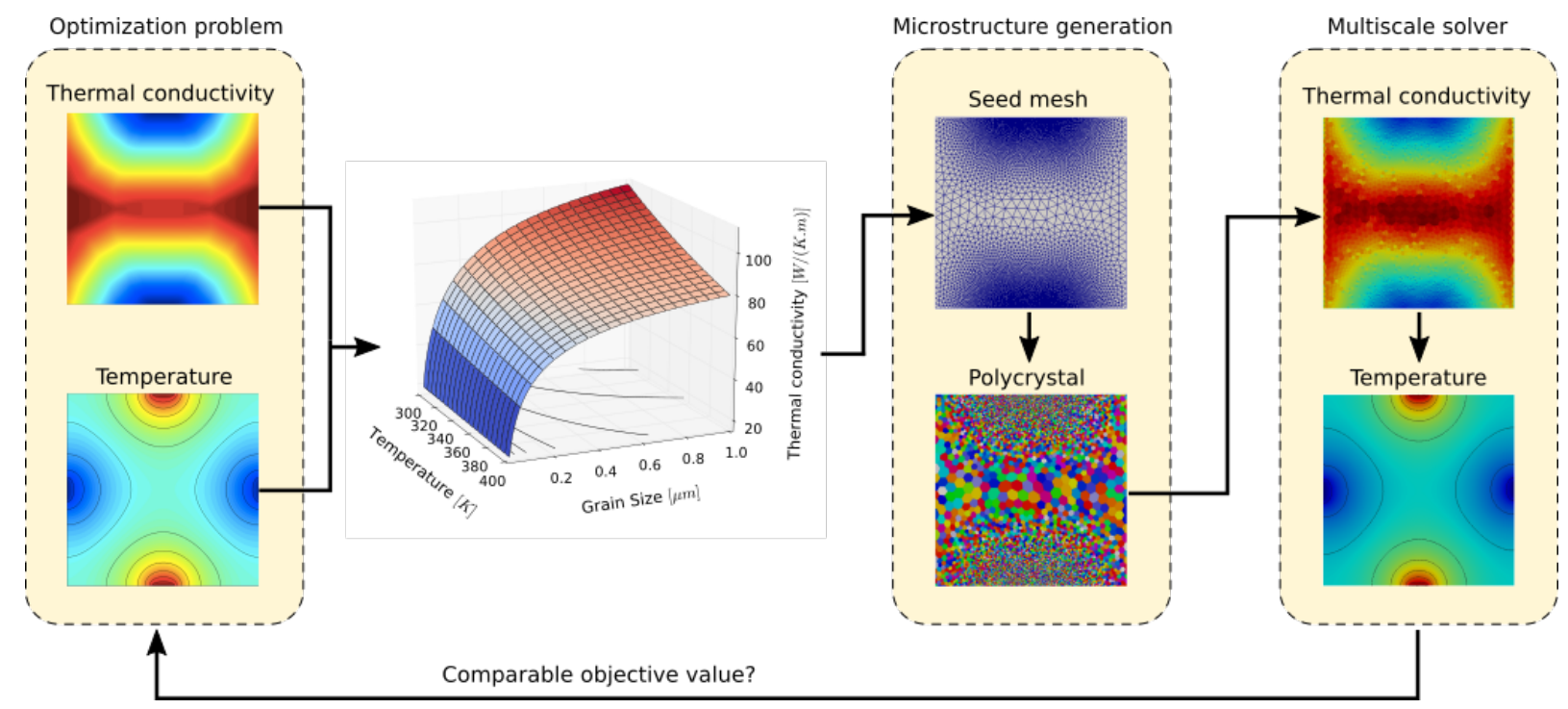

Figure 7: Schematics of procedure for verification of the separation of scales assumption.

computing the corresponding thermal solution, which is finally compared to the continuous one. The entire procedure, which starts with the results from the optimization problem, is depicted schematically in Fig. 7.

The microstructural reconstruction process is performed as follows. Utilizing the original computations of the attainable range of the thermal conductivity, we can proceed in reverse order: for a given control point with coordinates $X_{d}$ on the discrete mesh, we know the corresponding value of the optimal thermal conductivity $D_{d}$ and temperature $T_{d}$. Thus, by utilizing the temperature/grain-size/thermal conductivity relation as explained in section 2.2, we can infer the characteristic grain size $l_{d}$ at that point. Then, by adopting the same interpolation scheme as in the finite element calculations, we obtain the grain size field $l(X)$.

Given the grain size field, we generate a polycrystalline structure by following a procedure similar to the one proposed in [31], with the only difference that we adopt a dual based on the discrete Hodge operator [32] instead of a barycentric one. In this way, we entirely avoid the grain boundary energy relaxation step. This grain generation technique requires, as initial data, the location of the centers of each grain, which, in turn, should be spaced according to the required grain size distribution. As a result of this step, we obtain an explicit representation of the microstructure of the optimized material.

Once we generate the polycrystalline structure of the optimized material, we proceed to formulate a finite element model to solve for the corresponding thermal transport problem. The granular thermal conductivity is calculated at every quadrature point of the model 


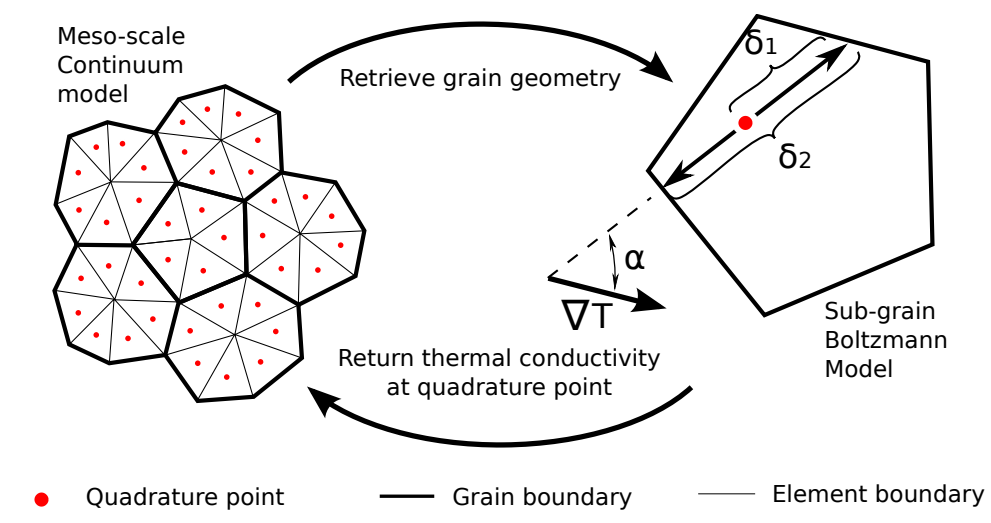

Figure 8: Schematics of concurrent multiscale scheme for meso-scale simulations.

by applying the procedure depicted in Subsection 2.1, see Fig. 8. Then, the Fourier heat conduction problem is solved. This procedure is nonlinear and requires an iterative process as the thermal conductivity depends on temperature. Convergence is generally obtained after a couple of iterations. This fast convergence is expected since the initial grain size distribution takes into consideration both the thermal conductivity and the temperature distribution at a point.

As a final step, the objective function is evaluated utilizing the previously obtained microstructural thermal distribution. The corresponding value of the objective function is evaluated and compared to the continuous temperature field resulting from the optimization problem. If results are not within required (problem specific) tolerances, it means that the separation of scales assumption does not hold for the specific problem under consideration. In that case, we should implement the optimization problem utilizing the real microstructure at each step, which would obviously incur a significantly higher computational cost.

\section{Results}

In this section, we illustrate the application of our method through two sample problems. In both problems, the full method, including mesh adaption and manufacturing constraints, is applied to optimize the thermal conductivity distribution within components. Sample problem \#1 focuses on the impact of limiting the grain size gradient and its effect on the objective function under consideration. Sample problem \#2 highlights the possible implications of grain size optimization by analyzing the relative impact of thermal conductivity optimization on a thermal barrier coating, as compared to the effect of a heat sink. In both of these problems, we optimize the grain size distribution for polycrystalline silicon, as all necessary material properties are broadly available in the literature. 


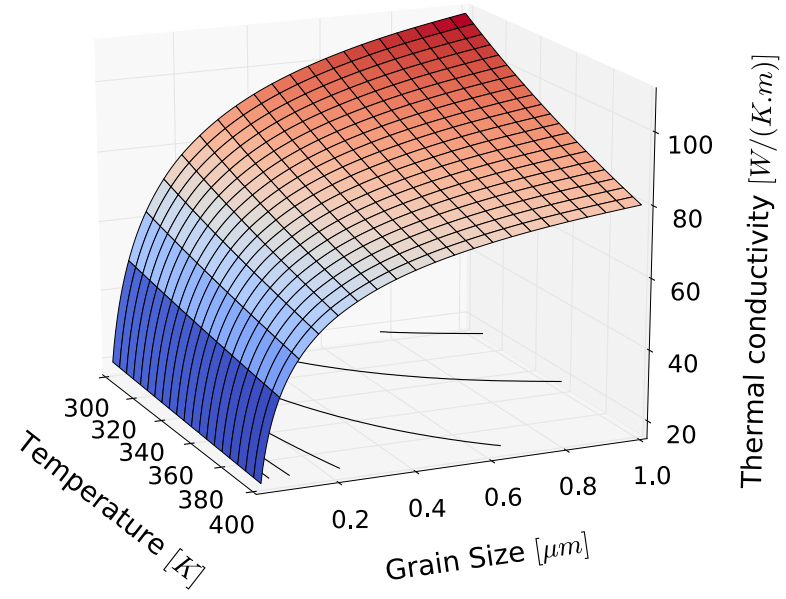

Figure 9: Thermal conductivity as a function of temperature and grain size.

The first step to approach both problems is to determine the attainable range of thermal conductivity, adopting the framework presented in section 2.1. Although this approach can potentially account for arbitrary grain size and shape, the latter determining thermal conductivity anisotropy, in this work we focus on the case of symmetric grains with isotropic thermal conductivity. That is, we focus solely on the effect of grain size and not on the impact of grain morphology. Thus, we assume non-elongated grains, shaped as regular hexagons. In order to remain within the scope of classical size-effects, we consider grain sizes within the range $l_{g} \in[0.5,20] \mu \mathrm{m}$. In agreement with boundary conditions for applications presented in rest of the paper, our study is limited to the temperature range $T \in[300,500] K$. The corresponding thermal conductivity obtained under these assumptions is depicted in Fig. 9. The attainable thermal conductivity range is then $D \in\left[40,110 \mathrm{~W} \mathrm{~K}^{-1} \mathrm{~m}^{-1}\right]$. It is worth noting that, even though only this range is needed for the optimization problem, the entire surface $F\left(T, l_{g}, D\right)=0$ must be kept in order to reconstruct the optimized microstructure in the verification step.

\subsection{Sample problem \#1}

This example evaluates the impact of the thermal conductivity gradient constraint on the optimization outcome. The problem under consideration is depicted by Fig. 10. The geometry is a square of length $L_{s}=200 \mu \mathrm{m}$. The bottom and top edges include constant temperature heat sources at $400 \mathrm{~K}$, while the right and left edges include heat sinks at $300 \mathrm{~K}$. Both heat sinks and sources are located at the center of their respective edges over $10 \%$ of the edge length. 


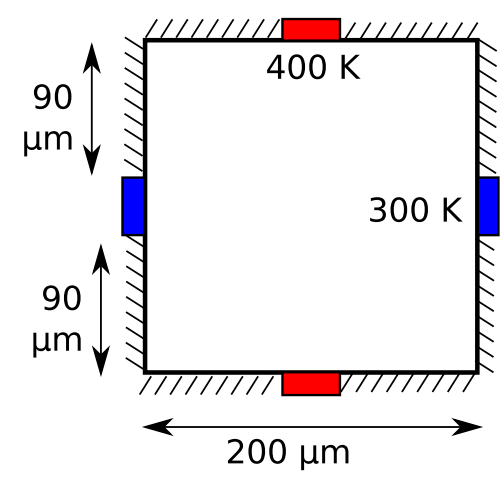

Figure 10: Geometry and boundary conditions of the sample problem \#1

The objective for this problem is to minimize the average temperature within the analysis domain. The corresponding optimization problem is expressed as

$$
\begin{array}{cl}
\underset{X_{d}, D_{d}}{\operatorname{minimize}} & \frac{1}{L_{s}^{2}} \int_{x \in\left[0, L_{s}\right]^{2}} T\left(x, X_{d}, D_{d}\right) d x \\
\text { subject to } & \nabla D_{\max }<\nabla D_{\text {opt }} \\
& X_{d} \in\left[0, L_{s}\right]^{N N} \\
& D_{d} \in[40,110]^{N}
\end{array}
$$

The optimal solution is expected to be a trade-off between isolating the heat sources with low thermal conductivity and connecting the heat sink to as much material with high thermal conductivity as possible. This balance is anticipated to be dependent on the thermal conductivity gradient constraint.

Several optimization problems are run with varying values of the non-dimensional parameter $\gamma \in[0,10]$, as defined by Eq. 22, to assess its effect on the optimal value of the objective function. A value $\gamma=0$ means no gradient on the thermal conductivity is allowed, or the equivalent assumption of constant thermal conductivity over the entire domain. It is worth noting that, since optimization is performed over the steady state solution of the thermal transport problem, the solution for the case with constant conductivity $(\gamma=0)$ is independent of the particular value of this material constant. The higher the value of $\gamma$, the higher the allowed maximum thermal conductivity gradient. Thus, in the limiting case $\gamma \rightarrow \infty$, there is no constraint on the gradient. This, in turn, allows very sharp contrasts on the thermal conductivity within the domain.

Results for varying values of the non-dimensional constant $\gamma$ are displayed in Fig. 11 . In the figure, blue circles represent the optimal solution for the continuous optimization 


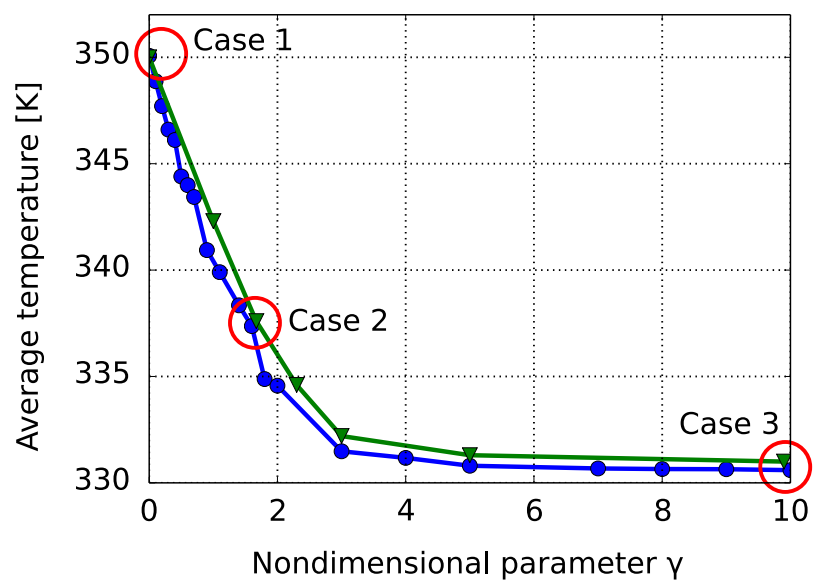

Figure 11: Influence of the thermal conductivity gradient on the optimal average temperature.

problem, whereas green triangles depict the value of the objective function for reconstructed microstructures (verification step). Notice that the verification step is performed on selected cases due to the computational expense associated with microstructural computations. The graph shows that, for the range considered in this example, the separation of scales assumption seems to be adequate.

As expected, a reduction of this constraint, i.e., larger values of $\gamma$, produces a better optimal solution. It is worth nothing, however, that while changes in performance are significant in the range $0 \leq \gamma \leq 3$, the effect is only minor for values $\gamma>3$. This indicates that, even though sharp solutions are ideal from a thermal performance perspective, a moderate level of manufacturing constraints does not affect much the overall performance of the system.

To conclude this sample problem, Fig. 12 depicts the reconstructed microstructures for representative cases 1,2, and 3, as highlighted on Fig. 11, along with their corresponding temperature distribution. As previously discussed, case (a) has a constant thermal conductivity in the entire domain. The average temperature for this case is $350 \mathrm{~K}$, as expected when considering the boundary conditions and configuration of the system. Case (b) is characterized by a value of the non-dimensional constant $\gamma=1.67$. The optimized configuration is defined by large grains around the heat sinks and small ones surrounding the heat source. That is, regions of large thermal conductivity connect heat sinks, while heat sources are isolated by means of areas with low thermal conductivity. The transition from large to small grain areas is smooth, as dictated by the gradient constrain. For this case, the optimal value of the objective function is $336.8 K$ and represents an improvement of $13.2 \%$ when compared to the constant thermal conductivity configuration. Finally, case (c) is almost free of any constraint, which generates a steep transition from regions with large 

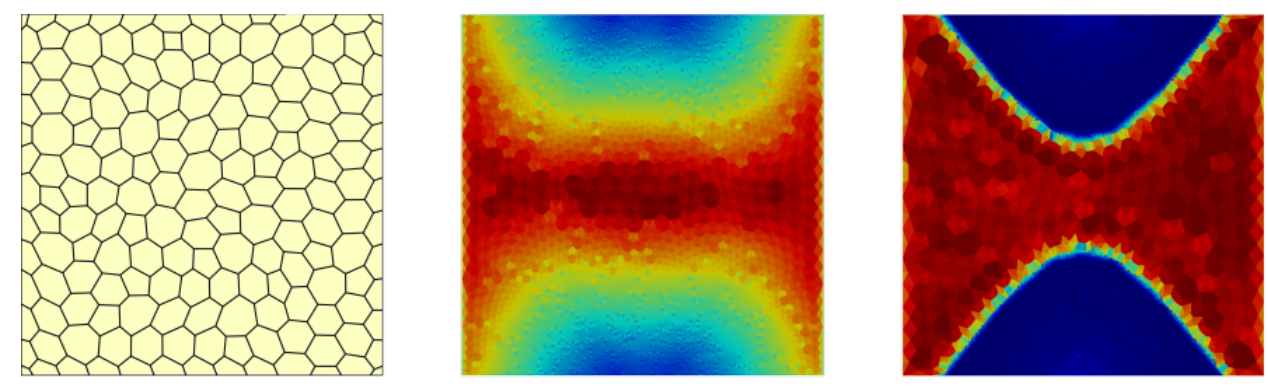

$\mathrm{D}[\mathrm{W} /(\mathrm{K} \cdot \mathrm{m})]$

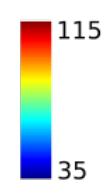

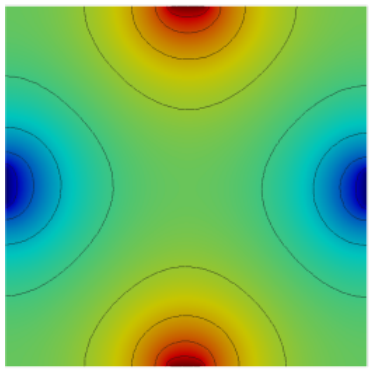

(a) Constant thermal conductivity

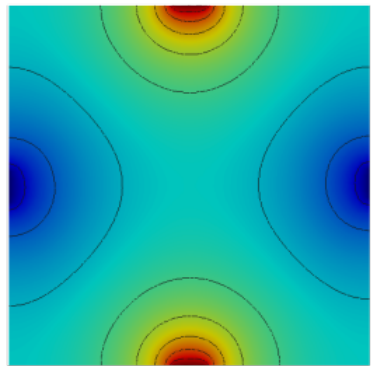

(b) Constrained gradient $\gamma=1.67$

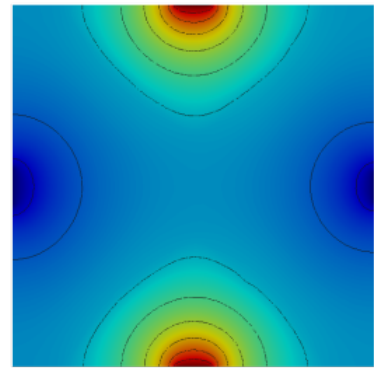

(c) Unconstrained optimization

Figure 12: Influence of thermal gradient constraint on temperature distribution.

to small grains. This configuration presents the best performance with an average temperature of $330.4 \mathrm{~K}$, equivalent to a $19.6 \%$ improvement with respect to the constant thermal conductivity case.

\subsection{Example 2}

In this final example of application, we focus on the optimization of a thermal barrier coating. The geometry and boundary conditions for this problem are depicted in Fig 13 . The geometry is a rectangle of height $400 \mu \mathrm{m}$ and width $200 \mu \mathrm{m}$. The bottom and top surfaces include heat sources at $400 K$ and $500 K$ respectively. The rectangle is split into 2 parts of equal height. The bottom part of the model, or substrate, consists of a constant thermal conductivity material, with $D=148 \mathrm{WK}^{-1} \mathrm{~m}^{-1}$ that corresponds to the bulk thermal conductivity of silicon. The top part, or coating, represents the design domain where the grain size is optimized. In this region, there is also a cooling pipe (heat sink) at a constant temperature $T=300 \mathrm{~K}$. The objective for this problem is to minimize the maximum temperature $T$ at the coating/substrate interface.

To better illustrate the effect of grain size optimization, we analyze three different configurations for this problem. In the first configuration, or case (a), we assume there is no cooling pipe. For this problem, we assign a constant value of thermal conductivity 


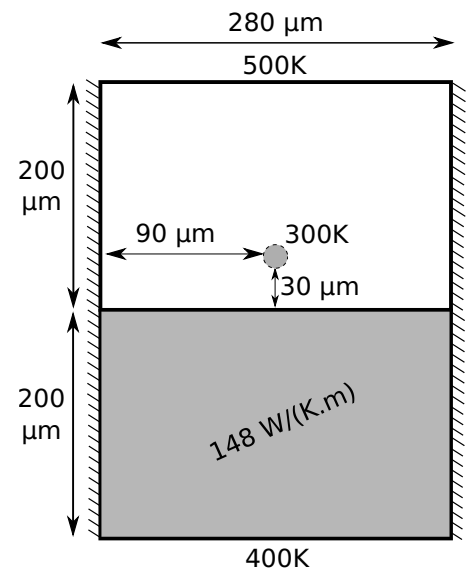

Figure 13: Geometry and boundary conditions for sample problem \#2.

$D$ over the coating domain. For the second configuration, or case (b), the same thermal conductivity $D$ is assigned to the top coating. In addition, we incorporate the cooling pipe into the system. Finally, in case (c), we consider a system where in addition to the presence of a cooling pipe, the thermal conductivity is optimized over the coating domain. To guarantee consistency among the different scenarios, we assign coating thermal conductivity $D=62.6 \mathrm{WK}^{-1} \mathrm{~m}^{-1}$ for cases (a) and (b), which is the same as the average thermal conductivity over the optimized domain for case (c).

For cases (a) and (b) there is no optimization to be performed, as we just need to solve for the corresponding temperature fields. For case (c), the optimization problem can be formulated as follows

$$
\begin{array}{cl}
\underset{X_{d}, D_{d}}{\operatorname{minimize}} & \max _{X_{d} \in\{X(x, y): 0 \leq x \leq 280, y=200\}} T\left(X_{d}, D_{d}\right) \\
\text { subject to } & \nabla D_{\max } \leq \nabla D_{\text {opt }}=175 \times 10^{3} W \cdot K^{-1} \cdot \mathrm{m}^{-2} \\
& X_{d} \in\left[0, L_{s}\right]^{2 N} \\
& D_{d} \in[40,110]^{N}
\end{array}
$$

The thermal conductivity distribution, as well as the corresponding optimal temperature field for each case is shown in Fig. 14. The maximum temperature at the interface for case (a) is $429.9 \mathrm{~K}$. The addition of the pipe on case (b) results in a temperature drop at the interface to a value of $388.2 \mathrm{~K}$. This significant improvement in the objective value -a $41.7 K$ decrease in maximum temperature at the coating/substrate interface- is expected and adopted as a reference to quantify the impact of the grain size optimization process. Finally, by optimizing the grain size distribution for case (c), we achieve a maximum temperature at the interface of $367.7 K$. This value represents a further $20.5 \mathrm{~K}$ 

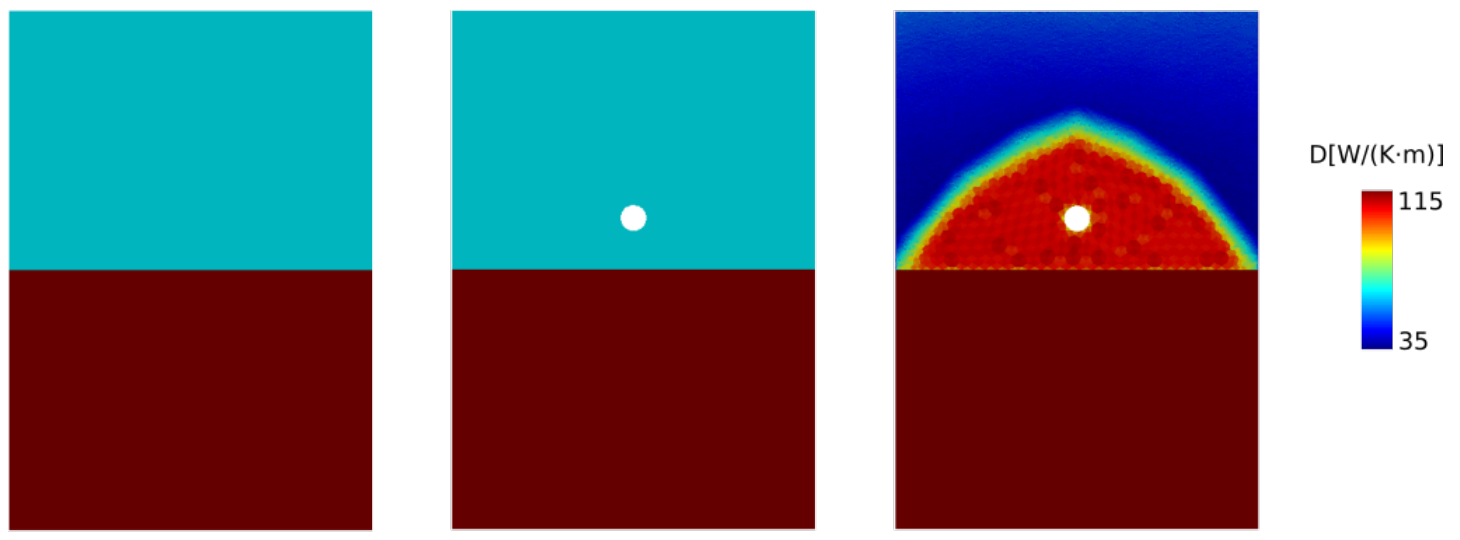

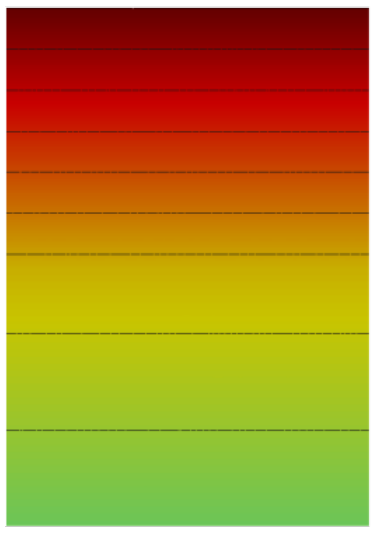

(a) Constant thermal conductivity - no pipe

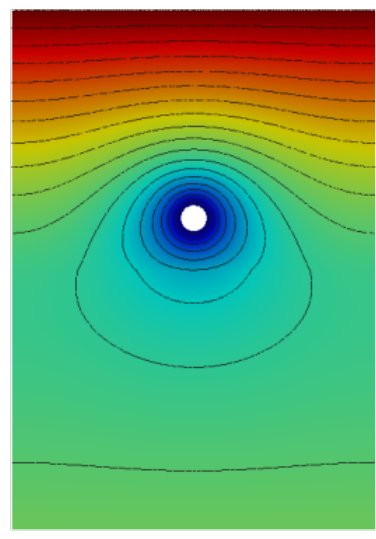

(b) Constant thermal conductivity - with pipe

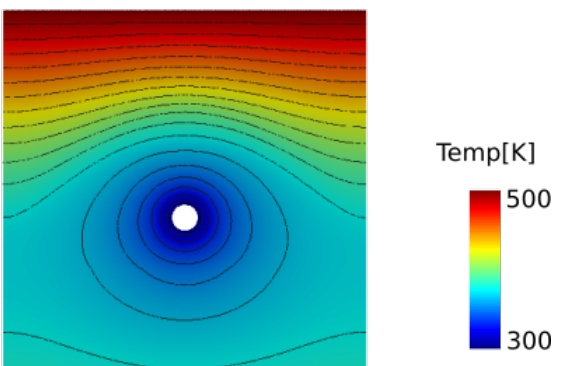

(3) Optimized grain size distribution - with pipe

Figure 14: Distribution of thermal conductivity and temperature field for three different configurations: constant thermal conductivity on coating (a), constant thermal conductivity and cooling pipe (b), optimal thermal conductivity distribution and cooling pipe (c).

temperature reduction at the interface. Thus, the benefit of the optimized grain size distribution represents $49.2 \%$ of the one provided by the pipe. That is, by optimizing the grain size distribution on the coating, it is possible to achieve a temperature reduction comparable to that obtained through the addition of a heat sink. Finally, note the overall temperature distribution is also remarkably different between the three different cases. It is worth remarking that this effect could be achieved with a single-material system, thus resulting in minimal thermal stresses associated to the variation in thermal conductivity. 


\section{Summary and concluding remarks}

Traditional approaches to thermal transport optimization usually rely on the presence of binary material system whose components exhibit a high contrast in the value of thermal conductivity. While such systems might result to be optimal from a thermal transport perspective, in general they would lead to significant thermal stresses at internal interfaces due to the associated high contrast in thermal expansion coefficients. These stresses, in turn, could lead to premature thermo-mechanical failure at internal interfaces. In this work, we proposed to circumvent this limitation by taking advantage of size-dependent effects in the thermal conductivity of materials. In our approach, we combined concurrent multiscale modeling with adaptive topology optimization techniques to optimize the spatial grain size distribution on single-material polycrystalline systems. We demonstrated that it is possible to develop single-material systems with optimized thermal transport properties.

\section{Acknowledgments}

This material is based upon work supported by the National Science Foundation Division of Civil, Mechanical, and Manufacturing Innovation under Grant No. CMMI-1454104.

\section{References}

[1] Martin Philip Bendsøe and Noboru Kikuchi. Generating optimal topologies in structural design using a homogenization method. Computer methods in applied mechanics and engineering, 71(2):197-224, 1988.

[2] Qing Li, Grant P Steven, Osvaldo M Querin, and YM Xie. Shape and topology design for heat conduction by evolutionary structural optimization. International Journal of Heat and Mass Transfer, 42(17):3361-3371, 1999.

[3] Qing Li, Grant P Steven, YM Xie, and Osvaldo M Querin. Evolutionary topology optimization for temperature reduction of heat conducting fields. International Journal of Heat and Mass Transfer, 47(23):5071-5083, 2004.

[4] Takayuki Yamada, Kazuhiro Izui, and Shinji Nishiwaki. A level set-based topology optimization method for maximizing thermal diffusivity in problems including designdependent effects. Journal of Mechanical Design, 133(3):031011, 2011.

[5] Katsuyuki Suzuki and Noboru Kikuchi. A homogenization method for shape and topology optimization. Computer methods in applied mechanics and engineering, 93(3):291-318, 1991. 
[6] George IN Rozvany, Ming Zhou, and Torben Birker. Generalized shape optimization without homogenization. Structural optimization, 4(3-4):250-252, 1992.

[7] Martin P Bendsøe and Ole Sigmund. Material interpolation schemes in topology optimization. Archive of applied mechanics, 69(9-10):635-654, 1999.

[8] YM Xie and Grant P Steven. A simple evolutionary procedure for structural optimization. Computers \&f structures, 49(5):885-896, 1993.

[9] Michael Yu Wang, Xiaoming Wang, and Dongming Guo. A level set method for structural topology optimization. Computer methods in applied mechanics and engineering, 192(1):227-246, 2003.

[10] Jan Stegmann and Erik Lund. Discrete material optimization of general composite shell structures. International Journal for Numerical Methods in Engineering, 62(14):2009-2027, 2005.

[11] Bouquet J. and Rimoli J. A length-dependent model for the thermomechanical response of ceramics. Journal of the Mechanics and Physics of Solids, 2015.

[12] A. McConnell and K. Goodson. Thermal Conduction in silicon micro- and nanostructures, volume 14, chapter II.3, pages 129-165. 2005.

[13] K. Goodson and M. Flik. Electron and phonon thermal conduction in epitaxial high-tc superconducting films. ASME Journal of Heat Transfer, 115:17-25., 1993.

[14] Jie Zou and Alexander Balandin. Phonon heat conduction in a semiconductor nanowire. Journal of Applied Physics, 89(5):2932-2938, 2001.

[15] M. Maldovan. Micro to nano scale thermal energy conduction in semiconductor thin films. Journal of Applied Physics, 110(3):034308, 2011.

[16] G. Tang, Y. Zhao, G. Zhai, and C. Bi. Phonon boundary scattering effect on thermal conductivity of thin films. J. Appl. Phys, 101:046102, 2011.

[17] Zhaojie Wang, Joseph E Alaniz, Wanyoung Jang, Javier E Garay, and Chris Dames. Thermal conductivity of nanocrystalline silicon: importance of grain size and frequency-dependent mean free paths. Nano letters, 11(6):2206-2213, 2011.

[18] John M Ziman, John Michael Ziman, John Michael Ziman, and Great Britain Physicist. Electrons and phonons: the theory of transport phenomena in solids. Clarendon Press Oxford, UK, 2001. 
[19] David G Cahill, Wayne K Ford, Kenneth E Goodson, Gerald D Mahan, Arun Majumdar, Humphrey J Maris, Roberto Merlin, and Simon R Phillpot. Nanoscale thermal transport. Journal of Applied Physics, 93(2):793-818, 2003.

[20] Charles Kittel and Paul McEuen. Introduction to solid state physics, volume 8. Wiley New York, 1976.

[21] Damian Terris, Karl Joulain, Denis Lemonnier, David Lacroix, and Patrice Chantrenne. Prediction of the thermal conductivity anisotropy of si nanofilms. results of several numerical methods. International Journal of Thermal Sciences, 48(8):14671476, 2009.

[22] Gang Chen. Thermal conductivity and ballistic-phonon transport in the cross-plane direction of superlattices. Physical Review B, 57(23):14958, 1998.

[23] G. Srivastava. The Physics of Phonons. Taylor \& Francis, New York, 1990.

[24] S. Adachi. Handbook on physical properties of semiconductors volume 1: Group-iv semiconductors. Kluwer Academic, 2004.

[25] Riccardo Tubino, Luigi Piseri, and Giuseppe Zerbi. Lattice dynamics and spectroscopic properties by a valence force potential of diamondlike crystals: C, si, ge, and sn. The Journal of Chemical Physics, 56(3):1022-1039, 1972.

[26] J. Turney, J. McGaughey, and C. Amon. In-plane phonon transport in thin films. Journal of Applied Physics, 107:024317, January 2010.

[27] Ercan M Dede. Simulation and optimization of heat flow via anisotropic material thermal conductivity. Computational Materials Science, 50(2):510-515, 2010.

[28] Garret N Vanderplaats. Multidiscipline design optimization. Vanderplaats Research \& Development, Incorporated, 2007.

[29] Warren Hare, Julie Nutini, and Solomon Tesfamariam. A survey of non-gradient optimization methods in structural engineering. Advances in Engineering Software, 59:19-28, 2013.

[30] P Thoutireddy and M Ortiz. A variational r-adaption and shape-optimization method for finite-deformation elasticity. International Journal for Numerical Methods in Engineering, 61(1):1-21, 2004. 
[31] JJ Rimoli and M Ortiz. A duality-based method for generating geometric representations of polycrystals. International Journal for Numerical Methods in Engineering, 86(9):1069-1081, 2011.

[32] R Hiptmair. Discrete hodge operators: An algebraic perspective. Progress In Electromagnetics Research, 32:247-269, 2001. 